\title{
hTERT-based therapy: A universal anticancer approach (Review)
}

\author{
MU-HAN LÜ ${ }^{1}$, ZHONG-LI LIAO ${ }^{1}$, XIAO-YAN ZHAO ${ }^{1}$, YA-HAN FAN ${ }^{1}$, XIAN-LONG LIN ${ }^{1}$, \\ DIAN-CHUN FANG ${ }^{2}$, HONG GUO $^{1}$ and SHI-MING YANG ${ }^{1}$ \\ ${ }^{1}$ Department of Gastroenterology, Xinqiao Hospital, Third Military Medical University, Chongqing 400037; \\ ${ }^{2}$ Department of Gastroenterology, Southwest Hospital, Third Military Medical University, Chongqing 400038, P.R. China
}

Received July 5, 2012; Accepted August 22, 2012

DOI: $10.3892 / o r .2012 .2036$

\begin{abstract}
Human telomerase reverse transcriptase (hTERT) has been identified as a major protein involved in aberrant cell proliferation, immortalization, metastasis and stemness maintenance in a majority of tumors, yet it has little or no expression in normal somatic cells. During the past few years, the development of hTERT-based therapies such as immunotherapy, suicide gene therapy and small-molecule interfering therapy have become critical and specific for eradicating all types of cancer. Here, current knowledge regarding hTERT and its involvement in various cancers and its role as a target of cancer therapies are reviewed. Additionally, hurdles to new cancer therapy development and new therapeutic opportunities are described, along with areas that require further investigation.
\end{abstract}

\section{Contents}

1. Introduction

2. Structure, function and regulation of hTERT

3. hTERT-based immunotherapy

Correspondence to: Dr Shi-Ming Yang or Dr Hong Guo, Department of Gastroenterology, Xinqiao Hospital, Third Military Medical University, Xinqiao Street, Shapingba District, Chongqing 400037, P.R. China

E-mail: shimingyang@yahoo.com

E-mail: haojia2004@yahoo.com.cn

Abbreviations: ALT, alternative lengthening of telomeres; APC, antigen-presenting cells; CTLs, cytotoxic T lymphocytes; DCs, dendritic cells; EGCG, epigallocatechin-3-gallate; HLA, human leukocyte antigen; HTL, helper T lymphocytes; hTERT, human telomerase reverse transcriptase; LAMP-1, lysosome-associated membrane protein; MAP, multiple antigenic peptide; MART-1, melanoma antigen recognized by $\mathrm{T}$ cell-1; MHC, major histocompatibility complex; RISC, RNA induced silencing complex; RTs, reverse transcriptases; siRNA, small interfering RNAs; TAAs, tumor-associated antigens; TRAIL, tumor necrosis factor-related apoptosis-inducing ligand; Treg, regulatory $\mathrm{T}$ cell

Key words: cancer, human telomerase reverse transcriptase, telomerase, therapy
4. hTERT-based suicide gene therapy

5. hTERT-based agents that block hTERT expression and biogenesis

6. Conclusions and prospects

\section{Introduction}

Telomeres are the physical ends of chromosomes that are composed of tandemly repeated G-rich DNA sequences in humans and other vertebrates (1), but progression through replication cycles will lead to telomere-dependent pathways of cell cycle arrest, senescence and mortality (2). To circumvent this crisis, telomerase, a ribonucleoprotein complex, adds TTAGGG repeats to the ends of the chromosomes (2). Human telomerase reverse transcriptase (hTERT) is the catalytic subunit of telomerase and is involved in the rate-limiting step in the activation of telomerase (3). The presence of hTERT is obligatory for aberrant cell proliferation and immortalization in most tumors $(>85 \%)$ (4) and recent studies have found that cancer stem cells are also hTERT-positive; however, hTERT has little or no expression in normal somatic cells (4). Recently, Lü et al observed that hTERT also plays a key role in the metastatic progression of gastric cancer (5). This finding suggests that, although hTERT itself is not an oncogene, hTERT inhibition in humans appears to be a tumor suppressor mechanism in both early- and late-stage cancers (6). Moreover, in contrast to hTERT, the expression of the other two subunits of telomerase, human telomerase RNA (hTR) and human telomerase associated protein (TP1), did not parallel telomerase activity (7) and are independent of tumor stage and histology. Based on the above features, hTERT is therefore considered an ideal therapeutic target in human cancer (Fig. 1).

Currently, therapy of malignant tumors still consists mainly of surgery, radiotherapy, chemotherapy and combinations of these methods (8), and these approaches for eradicating malignant tumor cells have their own weaknesses. Recently, promising new hTERT-based therapies have been developed, such as immunotherapy, suicide gene therapy, and smallmolecule interfering therapy. These treatments are critical and specific for eradicating many cancer types. Therefore, hTERT has been chosen as a molecular drug target in human cancer with a broad therapeutic window.

This review summarizes recent advances in hTERT-based drug development, and explores the experimental challenges 
that need to be overcome to develop hTERT-based therapies to treat human cancers.

\section{Structure, function and regulation of hTERT}

hTERT is encoded by a single copy gene, mapped to chromosome $5 \mathrm{p} 15,33$. The gene consists of 16 exons and 15 introns spanning more than $37 \mathrm{~kb}$ (9). The protein encoded by hTERT is composed of 1132 amino acid residues (9) that contain seven functional motifs conserved among reverse transcriptases (RTs) and one telomerase specific motif. hTERT belongs to a group of RTs, which is the key determinant of telomerase activity. Introduction of hTERT into normal telomerase-negative human cells can prevent entry into senescence, thereby extending the replicative life span of these cells. Furthermore, Yu et al observed that, at the cellular level, transfection of hTERT into U2OS cells, an hTERT-negative malignant cell line, enabled telomerase activity and further promoted their invasive and metastatic potential (10). It has been reported that TERT controls stem cells via transcriptional regulation of a developmental program converging on the $\mathrm{Wnt} / \beta$-catenin signaling pathway (11). Sequence analysis revealed that the hTERT promoter also lacks the TATA and CAAT boxes, but contains binding sites for several transcription factors, including the oncogenes SP1 and c-Myc (12), and the suppressor genes HER2 and p53 (13). Furthermore, downregulation of hTERT expression was partially mediated through inhibition of the DNA methyltransferase and histone acetyltransferase activities of the hTERT promoter. Treatment with epigallocatechin-3-gallate (EGCG) and a prodrug of EGCG, which remodel chromatin structures of the hTERT promoter by reducing the level of acetyl-H3K9, acetyl-H4, and acetyl-H3 binding to the hTERT promoter and facilitate the binding of hTERT repressors to the hTERT regulatory region, result in inhibition of hTERT transcription (14).

Attempts to regulate hTERT were initiated soon after research into the structure and function of hTERT began; however, hTERT expression is subject to multiple stages of control. These factors may be critical regulators of hTERT, not only in cancer cells but also in normal cells and the combined action of these regulators will determine the final expression pattern of hTERT. Therefore, the development of drugs that critically and specifically target hTERT is essential for cancer treatment.

\section{3. hTERT-based immunotherapy}

Identification of hTERT as a good, universal, tumor-associated antigen. The concept of cancer immunotherapy is based on manipulating the host immune system to attack the cancer. However, initial tumor immunotherapy proved disappointing because of the lack of 'resistance' to tumors in many animal cancer models. This lack of tumor immunogenicity may not arise because the tumor does not spontaneously express antigens but because the self-antigens expressed by the tumors do not effectively stimulate naïve or activated $\mathrm{T}$ cells. Therefore, expression in differentiated, healthy somatic cells resulted in cognate tumor immune escape. Consequently, it has been a challenge for researchers to identify ideal tumor-associated antigen (TAA) for the immunotherapy of various tumors. An ideal TAA should have the following characteristics: i) expres- sion in most cancers to be broadly applied to many different types of cancer; ii) restriction to the tumor to avoid autoimmune reactions; iii) rare expression in normal, mature tissues so that tolerance is broken; iv) possession of an irreplaceable role in tumor progression to prevent tumor variance and deletion; v) inducement of a strong immune response to repress tumors; and vi) recognition by both major histocompatibility complex (MHC)-I and -II in a restricted fashion to elicit a $\mathrm{CD}^{+}$and $\mathrm{CD}^{+} \mathrm{T}$ lymphocyte response (15).

To date, hTERT is the TAA that is most consistent with the above criteria. Accumulated evidence based on both humans and mice has shown that the same endogenous hTERT antigenspecific CTL can induce the efficient lysis of tumor cells of different histological origins and types (16). hTERT peptidespecific CTLs may derive from the original T cell pool, and can be primed for antitumor responses by hTERT antigen induction repeatedly (17). Immune escape by downregulation of hTERT expression in tumors could also lead to progressive telomere shortening and tumor death. Therefore, hTERT should be an ideal tumor-associated antigen (Fig. 2).

Activation of cytotoxic T-lymphocytes by hTERT-pulsed dendritic cells (DCs). DCs are the most efficient antigenpresenting cells (APCs), and they have been extensively used to activate antitumor effector $\mathrm{T}$ and $\mathrm{B}$ lymphocytes by presenting TAAs, including previously unknown epitopes restricted in various MHC-II manners (18). In addition, they are capable of promoting the activation of natural killer (NK) cells, which also have substantial antitumor effects. During the past few years, many studies have shown that DCs pulsed with hTERT RNA, DNA and an adenoviral vector containing the hTERT gene (Ad-hTERT) result in the restoration of telomerase activity and give rise to a strong $\mathrm{CD} 8^{+}$cytotoxic T-lymphocyte (CTL) response that specifically eradicates autologous tumor cells (19). Interestingly, DCs transduced with rAd-hTERT do not induce autoimmunity in normal control because the hTERT protein found in normal tissues is below the threshold level found in malignant cells that are recognized and lysed by hTERT-specific CTLs (19). Another study demonstrated that human DCs transfected with chimeric hTERT/lysosomeassociated membrane protein (LAMP-1) mRNA are capable of stimulating a $\mathrm{CD} 4^{+} \mathrm{T}$-cell reaction. This reaction is required to stimulate and sustain an optimal $\mathrm{CD} 8^{+} \mathrm{CTL}$ reaction in vivo (20). Moreover, the combination with adjuvant may induce DC maturation and activation, which may also enhance the immune response. However, obstacles to the development of this therapy should be taken into account, including potential adenovirus toxicity, high level of neutralizing antibodies, the inability of DCs to migrate to the draining lymph nodes, and activation or suppression by regulatory T-cells (Treg) (21). Therefore, further research regarding engineered hTERTmodified DC vaccines must be performed to address the above problems.

Identification of hTERT-based peptide epitopes. Peptide-based vaccination against tumors has progressed significantly based on the observations that $\mathrm{CD} 8^{+} \mathrm{CTL}$ lyse TAA-expressing tumor cells from multiple tissues and that $\mathrm{CD}^{+}$helper T lymphocytes (HTLs) are also activated by peptides derived from TAAs in the presence of DCs loaded with cognate TAAs 


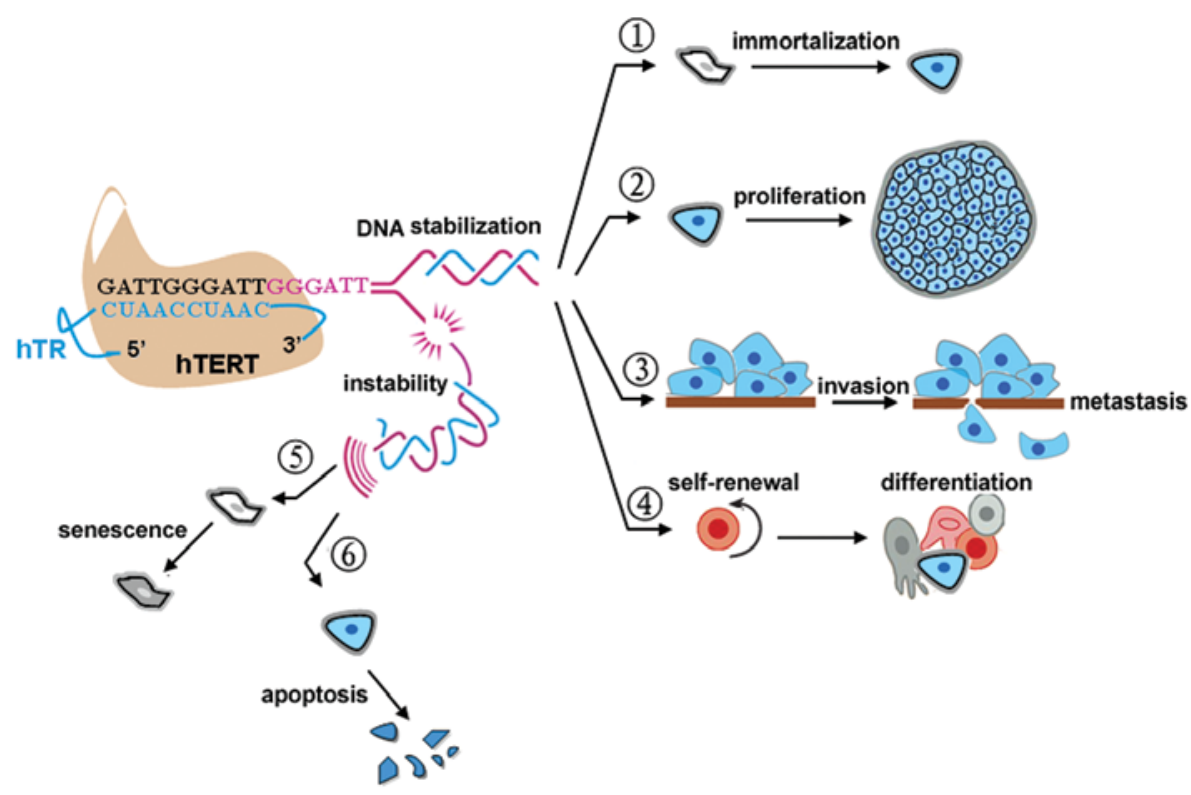

Figure 1. Characteristics of hTERT make it an ideal therapeutic target for human cancers. Aberrant overexpression of hTERT results in the following: 1, immortalization of primary human cells; 2 , enhancement of tumor cell proliferation; 3 , promotion of tumor invasive and metastatic potential; and 4, maintenance of stemness of stem cells, including self-renewal properties and pluripotency. Repression of hTERT results in telomere loss and the following: 5 , non-prevention of stress-induced senescence in normal cell; and 6, apoptosis of the tumor cell.

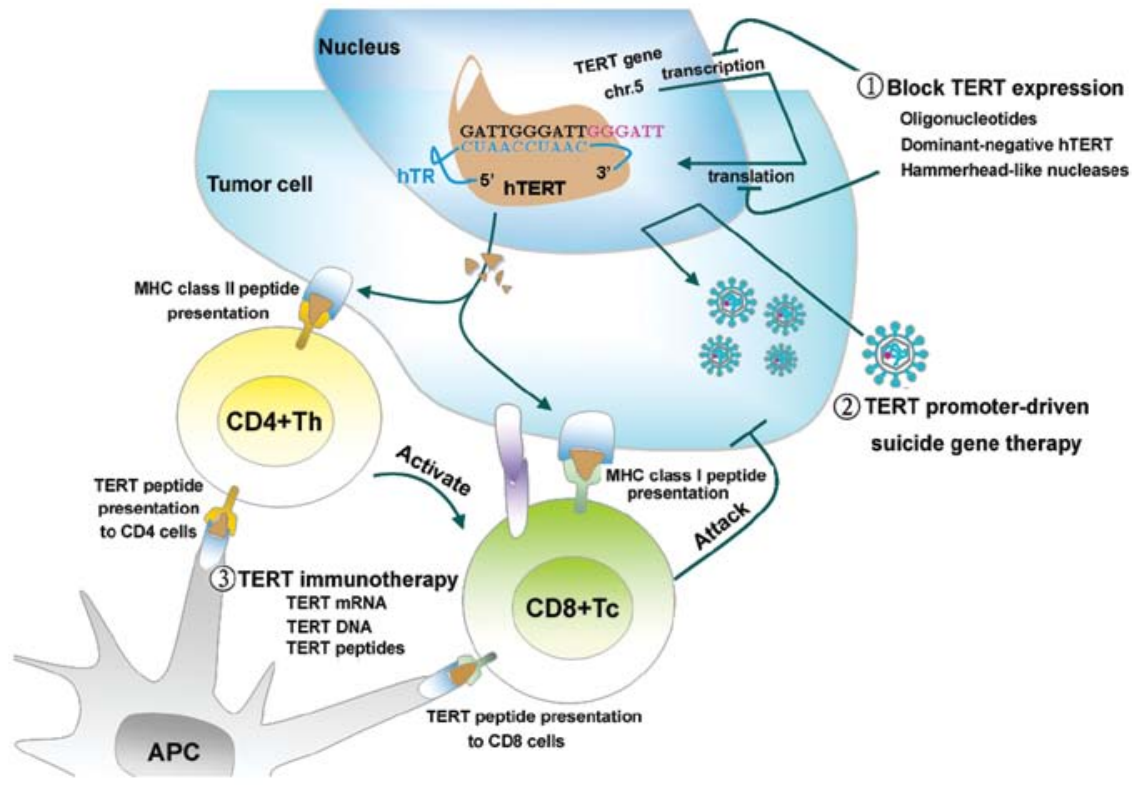

Figure 2. Three hTERT-based strategies for killing tumor cells. The presence of hTERT, as the rate-limiting step in the activation of telomerase, is a prerequisite for carcinogenesis. Recent evidence has shown that the recently developed hTERT-based therapies are successful in cancer treatment. 1, Studies regarding the regulation of hTERT were initiated soon after research into the transcription, translation, transport processing of hTERT was begun. These methodologies have the common goal of downregulating hTERT expression at multiple stages of biogenesis. Examples of these approaches include antisense oligonucleotides, dominant-negative hTERT, and hammerhead-like nucleases. 2, hTERT promoter-driven suicide gene therapy is based on genes that encode proteins that control the replication of microbial enzymes or oncolytic viruses that convert a prodrug into a toxic substance. This therapy is also based on effective delivery systems, such as liposomes and adenovirus vectors. 3, There is increasing evidence that the hTERT antigen recognized in both an MHC-I and -II restricted fashion elicits a $\mathrm{CD}^{+}$and $\mathrm{CD}^{+} \mathrm{T}$ lymphocyte response. In the immune response, DCs are the most efficient antigen-presenting cells. They have been used extensively to activate antitumor effector T and B lymphocytes through pulsing with hTERT RNA, DNA and an adenoviral vector containing the hTERT gene (Ad-hTERT) to specifically eradicate autologous hTERT-positive tumor cells. The relative advantages and disadvantages of these different methodologies are reviewed in this study.

(15). The I540 (ILAKFLHWL) was the first hTERT immunogenic peptide identified by epitope prediction from melanoma, and it has entered phase III clinical trials for melanoma treatment (22). To boost the anti-hTERT immune response, 38 hTERT peptides have been subsequently identified that are capable of inducing specific CTLs in vitro or in vivo (23-33) (Table I). The K973 peptide (KLFGVLRLK) (Table I) binds tightly to HLA-A3; it is capable of inducing a specific CTL response 
Table I. hTERT antigenic peptides identified for tumor immunity.

\begin{tabular}{|c|c|c|c|c|c|c|}
\hline Epitope & Sequence & Position & MHC & Cell line/in vivo & $\mathrm{CD} 4 / \mathrm{CD} 8$ & Refs. \\
\hline M1 & MPRAPRCRA & $1-9$ & HLA-B7 & $+/+\mathrm{M}$ & $-/+$ & $(29)$ \\
\hline R30 & RLGPQGWR & $30-37$ & HLA-A2 & $+/+\mathrm{M}$ & $-/+$ & $(26)$ \\
\hline A68 & APSFRQVSCL & $68-77$ & HLA-B7 & $+/+\mathrm{M}$ & $-/+$ & $(34)$ \\
\hline A167 & AYQVCGPPL & $167-175$ & HLA-A24 & $+/+\mathrm{H}, \mathrm{M}$ & $-/+$ & $(28)$ \\
\hline R277 & RPAEEATSL & $277-285$ & HLA-B7 & $+/+\mathrm{M}$ & $-/+$ & (29) \\
\hline V324 & VYAETKHFL & $324-332$ & HLA-A24 & $+/-$ & $-/+$ & $(29)$ \\
\hline Y325 & YLEPACAKY & $325-333$ & HLA-A1 & $+/+\mathrm{M}$ & $-/+$ & $(27)$ \\
\hline R342 & RPSFLLSSL & $342-350$ & HLA-B7 & $+/+\mathrm{M}$ & $-/+$ & $(29)$ \\
\hline R351 & RPSLTGARRL & $351-360$ & HLA-B7 & $+/+\mathrm{M}$ & $-/+$ & $(29)$ \\
\hline Y386 & YWQMRPLFLELLGNH & $386-400$ & HLA-DP & $+/-$ & $+/-$ & (32) \\
\hline D444 & DPRRLVQLL & $444-452$ & HLA-B7 & $+/+\mathrm{M}$ & $-/+$ & $(30)$ \\
\hline V461 & VYGFVRACL & $461-469$ & HLA-A24 & $+/-$ & $-/+$ & $(29)$ \\
\hline F464 & FVRACLRRL & $464-472$ & HLA-B7 & $+/+\mathrm{M}$ & $-/+$ & $(30)$ \\
\hline I540 & ILAKFLHWL & $540-548$ & HLA-A2 & $+/+\mathrm{H}, \mathrm{M}$ & $-/+$ & (23) \\
\hline L541 & LAKFLHWLMSVYVVE & $541-555$ & HLA-DP & $+/-$ & $+/-$ & $(31)$ \\
\hline L555 & LLRSFFYN & $555-563$ & HLA-A2 & $+/+\mathrm{M}$ & $-/+$ & (33) \\
\hline R572 & RLFFYRKSV & $572-580$ & HLA-A2 & $+/+\mathrm{M}$ & $-/+$ & $(24)$ \\
\hline L573 & LFFYRKSVWSKLQSI & $573-584$ & HLA-DP & $+/-$ & $+/-$ & $(31)$ \\
\hline E611 & EARPALLTSRLRFIPK & $611-626$ & HLA-DR, DQ, DP & $-/+\mathrm{H}$ & $+/-$ & $(31)$ \\
\hline R613 & RPALLTSRLRFIPKP & $613-627$ & HLA-DP & $+/-$ & $+/-$ & $(31)$ \\
\hline D637 & DYVVGARTF & $637-645$ & HLA-A24 & $+/+\mathrm{H}, \mathrm{M}$ & $-/+$ & $(28)$ \\
\hline A660 & $\begin{array}{l}\text { ALFSVLNYERARRPGLLGA } \\
\text { SVLGLDDIHRA }\end{array}$ & $660-689$ & HLA-A2, DR & $+/-$ & $+/+$ & (32) \\
\hline S663 & SVLNYERARRPGLLG & $663-677$ & HLA- DR & $+/-$ & $+/-$ & $(32)$ \\
\hline R672 & RPGLLGASVLGLDDI & $672-686$ & HLA-DR1, 7, 15 & $+/+\mathrm{M}$ & $+/-$ & $(35)$ \\
\hline P673 & PGLLGASVLGLDDIH & $673-687$ & HLA-A2, DR & $+/-$ & $+/+$ & $(32)$ \\
\hline G674 & GLLGASVLGL & $674-683$ & HLA-A2 & $+/-$ & $-/+$ & $(32)$ \\
\hline L766 & LTDLQPYMRQFVAHL & $766-780$ & HLA-DR1, 7, 15 & $+/+\mathrm{M}$ & $+/-$ & (36) \\
\hline C845 & CYGDMENKL & $845-853$ & HLA-A24 & $+/+\mathrm{H}, \mathrm{M}$ & $-/+$ & $(28)$ \\
\hline R865 & RLVDDFLLV & $865-873$ & HLA-A2 & $+/+\mathrm{H}, \mathrm{M}$ & $-/+$ & $(23)$ \\
\hline K973 & KLFGVLRLK & $973-981$ & HLA-A2, A3 & $+/-$ & $-/+$ & (34) \\
\hline D988 & DLQVNSLQTV & 988-997 & HLA-A2 & $+/+\mathrm{M}$ & $-/+$ & $(25)$ \\
\hline T1088 & TYVPLLGSL & $1088-1096$ & HLA-A24 & $+/+\mathrm{H}, \mathrm{M}$ & $-/+$ & $(28)$ \\
\hline L1107 & LPGTTLTAL & $1107-1115$ & HLA-B7 & $+/+\mathrm{M}$ & $-/+$ & (30) \\
\hline L1123 & LPSDFKTIL & $1123-1131$ & HLA-B7 & $+/+\mathrm{M}$ & $-/+$ & (30) \\
\hline$Y 572^{\mathrm{a}}$ & YLFFYRKSV & $572-580$ & HLA-A2 & $+/+\mathrm{M}, \mathrm{H}$ & $-/+$ & (25) \\
\hline$Y 988^{\mathrm{a}}$ & YLQVNSLQTV & 988-997 & HLA-A2 & $+/+\mathrm{M}$ & $-/+$ & $(25)$ \\
\hline$R 38^{\mathrm{a}}$ & RLGPQGWRV & $30-38$ & HLA-A2 & +/+M,H & $-/+$ & (26) \\
\hline
\end{tabular}

In cell line/in vivo column +, have test; -, no test; H, human test; M, mouse test; in CD4/CD8 column +, positive immune response; -, negative

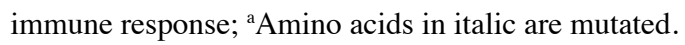

in an MHC-restricted manner, and it can also lyse a range of HLA-A2-positive tumor cell lines derived from various histological origins (34). HLA-A3, expressed in $15-25 \%$ patients, can expand the range of hTERT-based tumor immunotherapy to more than $60 \%$ of people. The peptide sequences VYAETKHFL and VYGFVRACL (Table I), derived from hTERT, are capable of eliciting hTERT peptide-specific CD8 ${ }^{+}$
CTLs that generate a cytotoxic response against leukemia cells in an HLA-A24-restricted manner (29). HLA-A24 is the most common allele and is present in more than $60 \%$ of Japanese and in nearly $20 \%$ of Europeans. These data strongly suggest that hTERT-based specific CTL responses for cancer immunotherapy can occur in most parts of the world. CD4 ${ }^{+}$ Th cells exert helper activity for initiating, regulating, and 
maintaining the $\mathrm{CD}^{+} \mathrm{CTL}$ response. Schroers et al identified two MHC-II restricted antigen peptides from hTERT, the L766 epitope (LTDLQPYMRQFVAHL) which incorporates to HLA-DR4, DR11, and DR15 and the R672 epitope (RPGLLGASVLGLDDI) which incorporates to HLA-DR1, DR7 and DR15 $(35,36)$ (Table I). These results illustrate that the binding of hTERT peptides is promiscuous; one Th epitope may be present within the different binding grooves of a single MHC-II molecule, and this attribute plays a role in the induction of a broader immune reaction. To enhance their ability to bind the MHC molecule, the majority of low-affinity peptides are modified with a tyrosine in the first position. This modification makes them highly immunogenic, and they can then induce a potent immune response for cancer treatment $(26,36)$.

Applications of hTERT-based peptides for cancer vaccines. It has not yet been determined whether these hTERT single epitope vaccines mediate an immunodominant response. This uncertainty may be due to their low molecular weight, rapid decline, weak immunogenicity, and their ineffective processing and presentation on cancer cells (37). To overcome the above problems, various carrier proteins and fusion proteins were used as vectors for delivering T-cell epitopes, while there is a risk that foreign proteins with high molecular weights will induce the immune response, rather than the target polypeptides. Currently, single-epitope peptide vaccines are based on HLA-restricted peptide predictive algorithms (38) and are used in patients with a particular HLA type, leading to a narrow therapeutic window, however, vaccinations with full-length mRNA encoding defined antigens or with multi-epitope, superimposed antigens have many known and unknown MHC-restricted epitopes.

hTERT peptides are processed and presented on the surface of DCs in the form of multiple-epitope that produce multiple CTL cell clones to induce an effective antitumor response using the MHC-I and -II pathways and avoid immune escape due to the loss of a single HLA allele. More attractively, the multiple antigenic peptide (MAP) system based on the core matrix lysine being coupled with four or eight strips of epitope monomer to form a branch-like structure, represents a unique way to generate anti-peptide antibodies (37). Theoretically, the MAP structure not only strengthens the specificity of the peptide chain structure and increases the molecular weight of the epitope peptides but also induces a high titer, high affinity antibody. Moreover, the Th epitopes are also added to the MAP system to improve vaccine immunogenicity and enhance CTL responses in an effective way. Recently, it has been reported that the synthetic dendritic tandem multiple antigenic hTERT epitope peptides consisting of I540 in a HLA-A02-restricted manner, V461 in a HLA-A24-restricted manner, and L766 in a HLA-DR-restricted manner are capable of inducing powerful antitumor responses in SW480/A549 cells expressing HLA-A2, HepG2/SMMC-7721 cells expressing HLA-A24, and SKOV3 cells negative for HLA-A2/A24 (39). The results also showed that the immunogenicity of the MAPs was better than a simple combination of the three individual peptides.

\section{4. hTERT-based suicide gene therapy}

Cancer gene therapy depends on an effective vector system and highly specific molecular targets. Currently, vector systems consist of non-viral vectors, such as naked DNA injections and liposomes, and viral vectors, such as adenovirus vectors. This methodology is based on genes that encode proteins that control the replication of microbial enzymes or oncolytic viruses that convert a prodrug into a toxic substance (40) (Fig. 2). Because the enzymatic or oncolytic virus systems can activate prodrugs only in the infected tumor cells, only the suicide gene-positive cells can be inhibited and even killed, while leaving normal somatic cells undamaged (40). Because of these advantages, adenovirus vector systems are commonly used in suicide gene therapy. The first generation of adenoviral vectors used to establish E1/E3-deletion constructs were not replication-competent, in order to be biologically safe. The increased interest in the field is expected because of the augmentation of transgene expression from the tumor-specific promoter without loss of target specificity. Jacob et al constructed Ad/TRAIL-F/RGD, the human tumor necrosis factor-related apoptosis-inducing ligand (TRAIL) gene under the transcriptional control of hTERT promoters. That report also demonstrated that treatment with Ad/TRAIL-F/RGD resulted in the apoptosis of human pancreatic and colon cancer cells in vitro, as well as in vitro suppression of tumor growth in an orthotopic implantation tumor model in the pancreas of mice, while having a minimal effect on normal cells (41). However, because the first generation of adenoviral vectors is not replication-competent, the treatment effect is confined only to the initially infected tumor cells, limiting the long-term therapeutic effect. Another concern is that the hTERT promoter activity with regard to targeted cancer gene therapy is low in stem cells (42), and the question remains as to whether this methodology can be applied to cancer stem cell therapy.

Although suicide gene therapy has made progress, the preclinical experimental results are still unsatisfactory. This method still cannot compensate for the shortcomings of replication-defective viruses in vivo, even if it is mediating strong bystander effects that affect not only the tumor cells transduced with the gene but also neighboring tumor cells. Therefore, conditionally replicating adenoviruses are emerging as a promising modality for cancer treatment. A novel replicative adenoviral vector, AdhTERTp-E1A, in which the hTERT promoter controls the selective replication of the vector in various tumor cells was reported. This attribute, combined with its lack of toxicity, can essentially promote oncolytic therapy as an antitumor treatment. Compared to the replication-defective virus, conditionally replicating adenoviruses under control of the hTERT promoter reduce tumor cell proliferation, play a more effective role in inhibiting tumor progress, are as safe as the former, and have no apparent side effects. Moveover, different hTERT promoters are used at different research institutions, various strategies have been devised to improve hTERT promoter activity. Several binding sites for transcription factors, including the transcription activating factors Myc and SP1, have been cloned into the adenoviral vector, and a series of constructs containing the E-box or TATA box upstream of the transcriptional start site have been cloned into the binding site for transcriptional activation of the hTERT gene (43). In addition, a single bicistronic adenoviral vector expressing pro-apoptotic genes under control of the hTERT promoter was constructed using the inducible gene expression system, providing a promising new systemic 
delivery vehicle for oncolytic adenoviruses. The novel vector utilizes the hTERT promoter to drive the expression of the transactivator that binds to the tetracycline-responsive element without tetracycline. This effect in turn causes the expression of the tumor-specific Bax gene in various types of tumors (44).

hTERT-based suicide gene therapy may rapidly kill hTERTpositive cells in tumors versus normal cells. However, efficient delivery of gene therapy to tumors throughout the human body is a major challenge, and immunological reactions may limit the dosing of the vector system.

\section{5. hTERT-based agents that block hTERT expression and biogenesis}

Although recent studies have screened different therapies targeting hTERT, an effective and specific agent used in clinical trials has not currently been found. During the past few years, various hTERT-based agents, including antisense oligonucleotides, overexpression of dominant-negative hTERT, and hammerhead-like nucleases, have been described (Fig. 2). Indeed, antisense oligonucleotide methodologies such as RNA interference were found efficient for hTERT gene silencing. The RNA interference is a natural process in which gene expression is silenced by small interfering RNAs (siRNA) that are complementary to the messenger RNA in eukaryotic cells. These siRNAs become incorporated into the RNA induced silencing complex (RISC) through a cleavage mechanism (45). Liu et al demonstrated that dendrimer-mediated shRNA against hTERT led to a marked reduction of hTERT expression in human oral cancer cells and mouse tumor xenografts (46). Gandellini et al showed that hTERT-specific siRNAs effectively impaired tumor cell growth and induced a variable degree of programmed cell death (47). Additionally, microRNAs, endogenous small RNAs, are hypothesized to be involved in the regulation of the hTERT gene (48). However, the relative efficacy and specificity of siRNAs needs to be carefully assessed for cell-type-dependent global effects and positional effects that may limit target accessibility by the siRNAs.

Hammerhead and hairpin ribozymes are attractive tools due to their small molecular size and the ease with which it is possible to design these molecules for use in the gene therapy field. Various studies have been performed regarding the use of hammerhead and hairpin ribozymes for cancer therapy. Hao et al designed a hammerhead anti-hTERT ribozyme and found that is exhibited both growth suppression and rapid apoptosis effects on an hTERT-positive carcinoma (49). Apoptosis of the cancer cells was not accompanied by telomere shortening, leaving no time for detecting replicative senescence. The induction of rapid apoptosis of cancer cells through the anti-hTERT ribozyme was via a direct mechanism rather than the telomere shortening-senescence-apoptosis pathway.

Telomerase inhibition in tumor cells using a dominantnegative hTERT mutant causes telomere shortening and tumor suppression. Sachsinger et al demonstrated that ectopic expression of a dominant-negative murine TERT mutant in renal tumor cells resulted in telomere shortening and telomerase inactivation (50).

Ribozymes, small interfering RNAs targeting TERT mRNA, and gene therapies using overexpressing mutant TERT showed good activity in some model systems. In addition to the ease of screening these potent candidate agents, another advantage is that these drugs directly and rapidly block hTERT expression and biogenesis. Specifically, antisense oligonucleotides are not removed from the cell by the efflux mechanisms responsible for the multidrug resistance of cancers (6). However, several obstacles such as effective transduction of these agents into cells without degradation and the avoidance of target-off effects for safety reasons need to be overcome before these anticancer therapies can be used for treatment.

\section{Conclusions and prospects}

Targeting hTERT is a promising approach in cancer treatment; however, there are many more opportunities and challenges ahead. Although all anti-hTERT therapies force telomere crisis, resulting in gradual cell apoptosis; however, tumors continue to grow during the telomere-shortening time. The prognosis of patients with cancer varies and may be dependent on telomerase activity, telomere length, and even the microenvironment of the tumor mass that can produce certain signals that decrease the effect of anti-cancer therapies (6). Furthermore, we need to note that the alternative lengthening of telomeres (ALT) mechanism represents a telomeraseindependent, recombination-based marker, referred to as ALT-associated tumor-type, to maintain telomere length (51). Therefore, targeting hTERT most likely needs to be coupled with other traditional therapeutic modalities to create new treatment strategies that lead to wider and more long-lasting response in cancer patients.

Currently, we remain at the early stages of clinical development of hTERT as a cancer target. The current hTERT-based drugs in preclinical and clinical trials are most likely not the best hTERT-based drugs that we can ultimately develop. The development of hTERT-based therapy provides an important new strategy for anticancer treatment; however, preclinical trials are needed to determine the dosage, time course of treatment, indications, and possible side effects of this promising anticancer treatment before entering into clinical use (15). hTERT-based therapies will be an inevitable part of cancer treatments in the near future.

\section{Acknowledgements}

This study was supported by the Natural Science Foundation of China (No. 81071845), the Chongqing Science Fund for Distinguished Young Scholars (CSTC, 2009BA5045) and the National Program for Key Basic Research Projects of China (973 Program, No. 2010CB529406).

\section{References}

1. Cohen SB, Graham ME, Lovrecz GO, Bache N, Robinson PJ and Reddel RR: Protein composition of catalytically active human telomerase from immortal cells. Science 315: 1850-1853, 2007.

2. Campisi J, Kim SH, Lim CS and Rubio M: Cellular senescence, cancer and aging: the telomere connection. Exp Gerontol 36: 1619-1637, 2001.

3. Zvereva MI, Shcherbakova DM and Dontsova OA: Telomerase: structure, functions, and activity regulation. Biochemistry 75 : $1563-1583,2010$. 
4. Raptis S and Bapat B: Genetic instability in human tumors. EXS 303-320, 2006

5. Lü MH, Deng JQ, Cao YL, Fang DC, Zhang Y and Yang SM: Prognostic role of telomerase activity in gastric adenocarcinoma: a meta-analysis. Exp Ther Med 3: 728-734, 2012

6. Harley CB: Telomerase and cancer therapeutics. Nat Rev Cancer 8: 167-179, 2008

7. Onoda N, Ogisawa K, Ishikawa T, Takenaka C, Tahara H, Inaba M, Takashima T and Hirakawa K: Telomerase activation and expression of its catalytic subunits in benign and malignant tumors of the parathyroid. Surg Today 34: 389-393, 2004

8. Sorbe B, Bohr L, Karlsson L and Bermark B: Combined external and intracavitary irradiation in treatment of advanced cervical carcinomas: predictive factors for local tumor control and early recurrences. Int J Oncol 36: 371-378, 2010.

9. Shay JW and Wright WE: Implications of mapping the human telomerase gene (hTERT) as the most distal gene on chromosome 5p. Neoplasia 2: 195-196, 2000.

10. Yu ST, Chen L, Wang HJ, Tang XD, Fang DC and Yang SM hTERT promotes the invasion of telomerase-negative tumor cells in vitro. Int J Oncol 35: 329-336, 2009.

11. Park JI, Venteicher AS, Hong JY, Choi J, Jun S, Shkreli M, Chang W, Meng Z, Cheung P, Ji H, et al: Telomerase modulates Wnt signalling by association with target gene chromatin. Nature 460: 66-72, 2009 .

12. Kanzawa T, Komata T, Kyo S, Germano IM, Kondo Y and Kondo S: Down-regulation of telomerase activity in malignant glioma cells by p27 ${ }^{\mathrm{KIPl}}$. Int J Oncol 23: 1703-1708, 2003.

13. Papanikolaou V, Iliopoulos D, Dimou I, Dubos S, Tsougos I, Theodorou K, Kitsiou-Tzeli S and Tsezou A: The involvement of HER 2 and $p 53$ status in the regulation of telomerase in irradiated breast cancer cells. Int J Oncol 35: 1141-1149, 2009.

14. Meeran SM, Patel SN, Chan TH and Tollefsbol TO: A novel prodrug of epigallocatechin-3-gallate: differential epigenetic hTERT repression in human breast cancer cells. Cancer Prev Res 4: 1243-1254, 2011

15. Zhang YF, Tang XD, Gao JH, Fang DC and Yang SM: Heparanase: a universal immunotherapeutic target in human cancers. Drug Discov Today 16: 412-417, 2011.

16. Amarnath SM, Dyer CE, Ramesh A, Iwuagwu O, Drew PJ and Greenman J: In vitro quantification of the cytotoxic T lymphocyte response against human telomerase reverse transcriptase in breast cancer. Int J Oncol 25: 211-217, 2004.

17. Titu LV, Loveday RL, Madden LA, Cawkwell L, Monson JR and Greenman J: Cytotoxic T-cell immunity against telomerase reverse transcriptase in colorectal cancer patients. Oncol Rep 12 871-876, 2004

18. Naito K, Ueda Y, Itoh T, Fuji N, Shimizu K, Yano Y, Yamamoto Y, Imura K, Kohara J, Iwamoto A, et al: Mature dendritic cells generated from patient-derived peripheral blood monocytes in one-step culture using streptococcal preparation OK-432 exert an enhanced antigen-presenting capacity. Int J Oncol 28: 1481-1489, 2006

19. Chen L, Liang GP, Tang XD, Chen T, Cai YG, Fang DC, Yu ST, Luo YH and Yang SM: In vitro anti-tumor immune response induced by dendritic cells transfected with hTERT recombinant adenovirus. Biochem Biophys Res Commun 351: 927-934, 2006.

20. Su Z, Vieweg J, Weizer AZ, Dahm P, Yancey D, Turaga V, Higgins J, Boczkowski D, Gilboa E and Dannull J: Enhanced induction of telomerase-specific CD4(+) T cells using dendritic cells transfected with RNA encoding a chimeric gene product. Cancer Res 62: 5041-5048, 2002.

21. Chen M, Chen G, Deng S, Liu X, Hutton GJ and Hong J: IFN-beta induces the proliferation of $\mathrm{CD}^{+}{ }^{+} \mathrm{CD} 25^{+} \mathrm{Foxp}^{+}$regulatory $\mathrm{T}$ cells through upregulation of GITRL on dendritic cells in the treatment of multiple sclerosis. J Neuroimmunol 242: 39-46, 2012.

22. Liu JP, Chen W, Schwarer AP and Li H: Telomerase in cancer immunotherapy. Biochim Biophys Acta 1805: 35-42, 2010.

23. Minev B, Hipp J, Firat H, Schmidt JD, Langlade-Demoyen P and Zanetti M: Cytotoxic $\mathrm{T}$ cell immunity against telomerase reverse transcriptase in humans. Proc Natl Acad Sci USA 97: 4796-4801, 2000.

24. Hernandez J, Garcia-Pons F, Lone YC, Firat H, Schmidt JD, Langlade-Demoyen $\mathrm{P}$ and Zanetti M: Identification of a human telomerase reverse transcriptase peptide of low affinity for HLA A2.1 that induces cytotoxic T lymphocytes and mediates lysis of tumor cells. Proc Natl Acad Sci USA 99: 12275-12280, 2002.
25. Scardino A, Gross DA, Alves P, Schultze JL, Graff-Dubois S, Faure O, Tourdot S, Chouaib S, Nadler LM, Lemonnier FA, et al: HER-2/neu and hTERT cryptic epitopes as novel targets for broad spectrum tumor immunotherapy. J Immunol 168: 5900-5906, 2002.

26. Thorn M, Wang M, Kloverpris H, Schmidt EG, Fomsgaard A, Wenandy L, Berntsen A, Brunak S, Buus S and Claesson MH: Identification of a new hTERT-derived HLA-A*0201 restricted, naturally processed CTL epitope. Cancer Immunol Immunother 56: 1755-1763, 2007.

27. Schreurs MW, Hermsen MA, Geltink RI, Scholten KB, Brink AA Kueter EW, Tijssen M, Meijer CJ, Ylstra B, Meijer GA and Hooijberg E: Genomic stability and functional activity may be lost in telomerase-transduced human $\mathrm{CD} 8^{+} \mathrm{T}$ lymphocytes. Blood 106: 2663-2670, 2005.

28. Mizukoshi E, Nakamoto Y, Marukawa Y, Arai K, Yamashita T, Tsuji H, Kuzushima K, Takiguchi M and Kaneko S: Cytotoxic $\mathrm{T}$ cell responses to human telomerase reverse transcriptase in patients with hepatocellular carcinoma. Hepatology 43: 1284-1294, 2006.

29. Adotevi O, Mollier K, Neuveut C, Cardinaud S, Boulanger E, Mignen B, Fridman WH, Zanetti M, Charneau P, Tartour E, et al: Immunogenic HLA-B*0702-restricted epitopes derived from human telomerase reverse transcriptase that elicit antitumor cytotoxic T-cell responses. Clin Cancer Res 12: 3158-3167, 2006

30. Cortez-Gonzalez X, Sidney J, Adotevi O, Sette A, Millard F, Lemonnier F, Langlade-Demoyen P and Zanetti M: Immunogenic HLA-B7-restricted peptides of hTRT. Int Immunol 18: 1707-1718, 2006.

31. Bernardeau K, Kerzhero J, Fortun A, Moreau-Aubry A, Favry E, Echasserieau K, Tartour E, Maillere B and Lang F: A simple competitive assay to determine peptide affinity for HLA class II molecules: a useful tool for epitope prediction. J Immunol Methods 371: 97-105, 2011

32. Suso EM, Dueland S, Rasmussen AM, Vetrhus T, Aamdal S Kvalheim G and Gaudernack G: hTERT mRNA dendritic cell vaccination: complete response in a pancreatic cancer patient associated with response against several hTERT epitopes. Cancer Immunol Immunother 60: 809-818, 2011.

33. Wang J, Yu L, Li J, Deng R and Wang X: Characterization of a human telomerase reverse transcriptase sequence containing two antigenic epitopes with high affinity for human leucocyte antigen. Biotechnol Appl Biochem 48: 93-99, 2007.

34. Vonderheide RH, Anderson KS, Hahn WC, Butler MO, Schultze JL and Nadler LM: Characterization of HLA-A3-restricted cytotoxic T lymphocytes reactive against the widely expressed tumor antigen telomerase. Clin Cancer Res 7: 3343-3348, 2001.

35. Schroers R, Huang XF, Hammer J, Zhang J and Chen SY: Identification of HLA DR7-restricted epitopes from human telomerase reverse transcriptase recognized by $\mathrm{CD} 4^{+} \mathrm{T}$-helper cells. Cancer Res 62: 2600-2605, 2002.

36. Schroers R, Shen L, Rollins L, Rooney CM, Slawin K, Sonderstrup G, Huang XF and Chen SY: Human telomerase reverse transcriptase-specific T-helper responses induced by promiscuous major histocompatibility complex class II-restricted epitopes. Clin Cancer Res 9: 4743-4755, 2003.

37. Tang XD, Wang GZ, Guo J, Lu MH, Li C, Li N, Chao YL, Li CZ, Wu YY, Hu CJ, Fang DC and Yang SM: Multiple antigenic peptides based on $\mathrm{H}-2 \mathrm{~Kb}$-restricted CTL epitopes from murine heparanase induce a potent antitumor immune response in vivo. Mol Cancer Ther 11: 1183-1192, 2012.

38. Parker KC, Bednarek MA and Coligan JE: Scheme for ranking potential HLA-A 2 binding peptides based on independent binding of individual peptide side-chains. J Immunol 152: 163-175, 1994.

39. Niu BL, Du HM, Shen HP, Lian ZR, Li JZ, Lai X, Wei SD Zou LQ and Gong JP: Myeloid dendritic cells loaded with dendritic tandem multiple antigenic telomerase reverse transcriptase (hTERT) epitope peptides: a potentially promising tumor vaccine. Vaccine 30: 3395-3404, 2012

40. Murofushi Y, Nagano S, Kamizono J, Takahashi T, Fujiwara H, Komiya S, Matsuishi T and Kosai K: Cell cycle-specific changes in hTERT promoter activity in normal and cancerous cells in adenoviral gene therapy: a promising implication of telomerasedependent targeted cancer gene therapy. Int J Oncol 29: 681-688, 2006.

41. Jacob D, Davis J, Zhu H, Zhang L, Teraishi F, Wu S, Marini FC III and Fang B: Suppressing orthotopic pancreatic tumor growth with a fiber-modified adenovector expressing the TR AIL gene from the human telomerase reverse transcriptase promoter. Clin Cancer Res 10: 3535-3541, 2004. 
42. Kyo S and Inoue M: Complex regulatory mechanisms of telomerase activity in normal and cancer cells: how can we apply them for cancer therapy? Oncogene 21: 688-697, 2002.

43. Iliopoulos D, Satra M, Drakaki A, Poultsides GA and Tsezou A Epigenetic regulation of hTERT promoter in hepatocellular carcinomas. Int J Oncol 34: 391-399, 2009.

44. Gu J, Zhang L, Huang X, Lin T, Yin M, Xu K, Ji L, Roth JA and Fang B: A novel single tetracycline-regulative adenoviral vector for tumor-specific Bax gene expression and cell killing in vitro and in vivo. Oncogene 21: 4757-4764, 2002.

45. Zhao P, Wang C, Fu Z, You Y, Cheng Y, Lu X, Lu A, Liu N, $\mathrm{Pu}$ P, Kang C, Salford LG and Fan X: Lentiviral vector mediated siRNA knock-down of hTERT results in diminished capacity in invasiveness and in vivo growth of human glioma cells in a telomere length-independent manner. Int J Oncol 31: 361-368, 2007.

46. Liu X, Huang H, Wang J, Wang C, Wang M, Zhang B and Pan C: Dendrimers-delivered short hairpin RNA targeting hTERT inhibits oral cancer cell growth in vitro and in vivo. Biochem Pharmacol 82: 17-23, 2011 .
47. Gandellini P, Folini M, Bandiera R, De Cesare M, Binda M, Veronese S, Daidone MG, Zunino F and Zaffaroni N: Downregulation of human telomerase reverse transcriptase through specific activation of RNAi pathway quickly results in cancer cell growth impairment. Biochem Pharmacol 73: 1703-1714, 2007.

48. Kota SK and Balasubramanian S: Cancer therapy via modulation of micro RNA levels: a promising future. Drug Discov Today 15: 733-740, 2010

49. Hao ZM, Luo JY, Cheng J, Li L, He D, Wang QY and Yang GX: Intensive inhibition of hTERT expression by a ribozyme induces rapid apoptosis of cancer cells through a telomere length-independent pathway. Cancer Biol Ther 4: 1098-1103, 2005.

50. Sachsinger J, Gonzalez-Suarez E, Samper E, Heicappell R, Muller M and Blasco MA: Telomerase inhibition in RenCa, a murine tumor cell line with short telomeres, by overexpression of a dominant negative mTERT mutant, reveals fundamental differences in telomerase regulation between human and murine cells. Cancer Res 61: 5580-5586, 2001.

51. Cesare AJ and Reddel RR: Alternative lengthening of telomeres: models, mechanisms and implications. Nat Rev Genet 11: 319-330, 2010. 